\section{P1-248 ULTRA-PROCESSED FOOD CONTRIBUTES WITH HIGH PROPORTIONS OF THE CALORIC INTAKE: RESULTS FROM A POPULATION BASED SURVEY IN SÃO PAULO, BRAZIL}

doi:10.1136/jech.2011.142976e.40

E V Junior, A M de Carvalho, S Selem, A N Previdelli, M A de Castro, R M Fisberg, D M L Marchioni.* University of São Paulo, São Paulo, Brazil

Introduction Ultra-processed foods (UPF), a new classification proposed recently, comprise those foods prepared by the food, drink and associated industries, like soft drinks, snacks, etc, that intentionally are added salt, fat, sugar, preservatives and flavours and colours. They are usually energy-dense, and contain considerable quantities of added sugar, sodium, saturated or trans fats and little dietary fibre. Some of them are already associated with the increasing of the risk of obesity.

Objective To estimate the ultra-processed foods consumption among Brazilian individuals.

Methods A population-based survey that participated 273 adolescents, 436 adults and 385 elderly from São Paulo, Brazil, held in 2008. Dietary intake was collected by the one $24 \mathrm{~h}$ recall. It was calculated the caloric share of this group in relation to total energy intake. Further, it was verified the foods with the greatest caloric share. The analyses were stratified by age group, sex, income and educational level.

Results The mean caloric share from UPF were 58\% (SD 21\%), 49\% (SD 24\%), and 42\% (22\%) for adolescents, adults and elderly, respectively $(p<0.001)$. The highest educational level was associated with higher caloric share from UPF for all age group and sexes. There were no differences among quartiles of income. The overall UPF with the greatest caloric share were bread (22\%), sandwiches and pizza (13\%), soft drink (7\%), biscuits (5\%), and sausage (4.5\%).

Conclusion The UPF consumption represents a large percentage of total caloric intakes, mainly in adolescents.

\section{P1-249 ADOPTION OF HOME SMOKING BANS AFTER THE IMPLEMENTATION OF NATIONAL SMOKE-FREE LEGISLATION. FINDINGS FROM THE INTERNATIONAL TOBACCO CONTROL (ITC) EUROPE SURVEYS}

doi:10.1136/jech.2011.142976e.41

U Mons. ${ }^{*}$ German Cancer Research Center, Heidelberg, Germany

Objective To examine changes in prevalence and predictors of home smoking bans (HSB) among smokers in four European countries after the implementation of national smoke-free legislation.

Methods Two waves (pre- and post-legislation) of the International Tobacco Control (ITC) Europe Surveys, prospective panel studies conducted in Ireland, France, Germany and the Netherlands. Of 6396 smokers interviewed before implementation of a national smoke-free policy, 4632 (72.5\%) could be followed-up after the implementation and were included in the analyses. Multiple logistic regression models were computed in order to identify factors associated with the presence or adoption of HSB among smokers.

Results Most smokers had at least partial smoking restrictions in their home, but the proportions varied significantly between countries. After implementation of national smoke-free legislation, the share of smokers with a total HSB increased significantly in all four countries. Multiple logistic regressions indicated that having a young child in the household and supporting smoking bans in bars were important predictors of banning smoking completely at home. Prospective predictors of imposing a HSB between survey waves were planning to quit smoking, supporting a full bar smoking ban, and the birth of a child.
Conclusions The findings support that smoke-free legislation does not lead to more smoking in smokers' homes, which is further evidence against the claim of a displacement of smoking into the private home following the implementation of public smoking bans. On the contrary, the findings suggest that smoke-free legislation might even stimulate smokers to establish total smoking bans in their homes.

\section{P1-250 PUFA: AN INNOVATIVE INDEX TO MEASURE THE CONSEQUENCES OF UNTREATED DENTAL DECAY}

doi:10.1136/jech.2011.142976e.42

${ }^{1} \mathrm{~B}$ Monse, ${ }^{2} \mathrm{H}$ Benzian, ${ }^{3} \mathrm{R}$ Heinrich-Weltzien, ${ }^{4} \mathrm{C}$ Holmgren, ${ }^{4} \mathrm{~W}$ van $\mathrm{P}$ Helderman. ${ }^{*}{ }^{1}$ Fit for School Inc., Manila, The Philippines; ${ }^{2}$ The Health Bureau Ltd, Haversham, UK; ${ }^{3}$ WHO Collaborating Center for Prevention of Oral Disease, University Jena, Jena, Germany; ${ }^{4}$ Department of Global Oral Health, Radboud University, Nijmegen, The Netherlands

Introduction Untreated dental caries is a global public health problem. Only limited data are available on the clinical consequences of untreated dental caries because there is no measure to quantify the prevalence and severity of oral conditions resulting from untreated dental caries. The new PUFA index records the presence of severely decayed teeth with visible pulpal involvement $(\mathrm{P} / \mathrm{p})$, ulceration caused by dislocated tooth fragments $(\mathrm{U} / \mathrm{u})$, fistula $(\mathrm{F} / \mathrm{f})$ and abscess $(\mathrm{A} / \mathrm{a})$; capital letters are for permanent and lowercase letters are for deciduous teeth. The PUFA/pufa score is calculated cumulatively representing the number of teeth that meet the PUFA/pufa diagnostic criteria.

Methods Three examiners were trained in PUFA use to assess reproducibility. Fifty 6-yr-old children and forty-nine 12-yr-old children were examined for PUFA/pufa and reproducibility assessed by $\kappa$ calculation. Subsequent validation of the index in the 2006 Philippine National Oral Health Survey included 2030 6-yr-old and 2022 12-yr-old children using standard oral examination conditions (WHO Basic Methods $4^{\text {th }}$ Edition).

Results Inter-examiner reproducibility prior to the survey had a $\mathrm{K}$ value of 0.85 . During the survey, intra-examiner reproducibility varied between $\kappa$ values of $0.80-0.97$ in both age groups. The index was easy to use under field survey conditions. The prevalence of PUFA/pufa $>0$ was $85 \% / 56 \%$ (6-/12-yr-olds). The mean PUFA/pufa score was $3.5 / 1.2$ and $40 \% / 41 \%$ of decayed teeth had progressed to odontogenic infections (6-/12-yr-olds).

Conclusion The PUFA index complements classical caries indices with important information for epidemiologists and healthcare planners.

\section{P1-251 IRANIAN HEALTH PERCEPTION STUDY: A NATIONWIDE SURVEY}

doi:10.1136/jech.2011.142976e.43

A Montazeri, * J Sadighi, F Farzadi, S Omidvari, F Maftoon, A Aeenparast, K Jahangiri, M Vahdaninia. Iranian Institute for Health Sciences Research, ACECR, Tehran, Iran

Introduction People's health perception could be important factor in understanding how they think and how they behave. This study thought to assess health perceptions among Iranians inorder to contribute to health policy in Iran.

Methods This was a nationwide cross sectional study. A random sample of individuals aged between 18 and 65 were entered into study. A designed questionnaire containing items on demographic and health-related behaviours (smoking, physical activity, nutrition), chronic diseases, importance of health, selfreported psychosocial and overall health status, and health 
information was used to collect data. Data were analysed in a descriptive fashion.

Results In all 27883 individuals were studied. The mean age of respondents was 32.7 ( $\mathrm{SD}=11.8$ ) years. Overall 25.4\% were smoker, $53.8 \%$ indicated that never or a little did exercise, $52.7 \%$ indicated that they were very or very much concerned about their nutrition; $20.1 \%$ reported high level of anxiety, $25.6 \%$ reported a degree of depression, 30\% stated that they did not care about their health, $79.3 \%$ reported a relatively high level of relationships with family, relatives and friends, $20.7 \%$ reported that they suffer from at least one chronic disease, and $27.9 \%$ reported less than good health status. Only $36.7 \%$ of the respondents said that they have enough information about health.

Conclusion Considering current healthcare programs in Iran it seems that policy makers should have a better understanding of people's health-related attitudes and behaviours.

\section{P1-252 CREUTZFELDT-JAKOB DISEASE (CJD) SURVEILLANCE IN TEXAS2010: A SURVEILLANCE ODYSSEY}

doi:10.1136/jech.2011.142976e.44

\section{K Moody.* Texas Department of State Health Services, Austin, Texas, USA}

Creutzfeldt-Jakob Disease (CJD) is a rare, invariably fatal neurodegenerative disease caused by misfolded proteins called prions. The disease can originate in three different ways (sporadic, genetic and infectious) and has four forms (sporadic, variant, iatrogenic, and familial). Known infectious routes include ingestion of contaminated beef (mad cow disease or vCJD), and certain surgical procedures and contaminated surgical instruments (iCJD). CJD is a difficult disease to diagnose; ultimately requiring neuropathological examination of brain tissue for confirmation. Clinical history, MRI, electroencephalogram and cerebrospinal fluid testing assessing 14-33 and Tau proteins levels are all tools that are used to assess the likelihood of CJD. Unlike most reportable conditions, CJD can only be confirmed after death. Because of the complexity of the disease, surveillance and case investigation can be a challenge. The average rate of CJD in the US is 1 case per million. Historically, Texas has averaged 16 cases per year (0.7 cases per million). Last year, 2010, was an exception. Not only did Texas have over 25 cases, 7 of these cases had an uncharacteristic clinical course. This presentation will take a historical look at CJD surveillance in Texas from 2000 to 2010 and will highlight the distinctiveness of 2010 .

\section{P1-253 LONG-TERM HEALTH RISKS AFTER INFECTIVE GASTROENTERITIS: A POPULATION-BASED COHORT STUDY}

doi:10.1136/jech.2011.142976e.45

\footnotetext{
${ }^{1,2} \mathrm{R}$ Moorin, ${ }^{*} \mathrm{~J}$ Heyworth, ${ }^{3} \mathrm{G}$ Forbes, ${ }^{2} \mathrm{~T}$ Riley. ${ }^{1}$ Curtin University, Western Australia, Australia; ${ }^{2}$ The University of Western Australia, Western Australia, Australia; ${ }^{3}$ Royal Perth Hospital, Western Australia, Australia
}

Introduction Gastroenteritis presents a considerable burden on communities and health systems. In addition to the immediate health consequences long-term adverse sequelae have been associated with gastroenteritis; however, the risk and type of sequelae attributable to previous exposure to enteric infections have not been quantified. The objective of this study was to quantify the risk and type of sequelae attributable to previous exposure to enteric infections occurring in childhood and adolescence.

Methods This was a population-based retrospective cohort study comprising all individuals born in Western Australia between 1 January 1985 and 31 December 2000 using linked administrative records from the Western Australian notifiable infectious diseases database, the Western Australian hospital morbidity data system and Western Australian death notifications. The risk of first-time hospitalisation for sequelae for those exposed to an enteric infection was modelled using Cox proportional regression analysis controlling for other health and socio-demographic factors.

Results After adjusting for confounding there was a significant increase in the rate of first-time hospitalisation for sequelae in those exposed to enteric infections by $64 \%$ for any sequelae; $52 \%$ for intragastrointestinal sequelae and $63 \%$ for extra-gastrointestinal sequelae compared with non-exposed individuals. This equated to an increased attributable risk of 39\% for all sequelae, 34\% for intragastrointestinal and 39\% for extra-gastrointestinal sequelae.

Conclusion Infective gastroenteritis in childhood or adolescence increases the risk of first-time hospitalisation for intra-gastrointestinal and extra-gastrointestinal disease over the following 2 decades. This highlights the importance of developing appropriate risk management strategies for those exposed to enteric infections.

\section{P1-254 DETERMINANTS OF REFUSALS IN A FOLLOW-UP EVALUATION OF A PORTUGUESE BIRTH COHORT}

doi:10.1136/jech.2011.142976e.46

1,2 V Morais, ${ }^{*}{ }^{1,2} \mathrm{~S}$ Correia, ${ }^{1,2} \mathrm{~A}$ C Santos, ${ }^{1,2} \mathrm{H}$ Barros. ${ }^{1}$ Department of Hygiene and Epidemiology, University of Porto Medical School, Porto, Portugal; ${ }^{2}$ Institute of Public Health, University of Porto, Porto, Portugal

Introduction The main advantages of birth cohort studies may be compromised by the refusals in participation, which tend to increase in time.

Objective To describe the main predictors of refusals in the Portuguese birth cohort 4 years after the assembling.

Methods Geração XXI was assembled in 2005/2006 in five public maternities in Porto Metropolitan area, Portugal. After delivery, data were collected by personal interviews. Sub-samples of mothers were re-assessed at 6, 15 and 24 months and an ongoing evaluation of the entire cohort is being performed at the age of $4 / 5$ years. Refusals at follow-up were compared with evaluated participants considering baseline maternal and childhood characteristics, fitting logistic regression models were adjusted for age, education level and working condition, place of residence and previous follow-ups.

Results Within 6025 participants already contacted, 88\% were evaluated and $704(12 \%)$ refused. The major determinant of refusal was the absence of previous evaluations (OR $=2.01 ; 95 \%$ CI 1.68 to 2.41). Refusals were more frequent among less educated women ( $\leq 9$ vs $\geq 13$ years of education: $\mathrm{OR}=1.23$; $95 \% \mathrm{CI} 1.01$ to 1.53 ), as within housewives (vs employed $\mathrm{OR}=1.60 ; 95 \%$ CI 1.14 to 2.25 ). No statistical significant differences were found regarding other maternal, birth or newborn characteristics. The main reasons for refusal were unavailability (39.6\%), personal/professional reasons (18.9\%) and distance to evaluation setting (16.1\%).

Conclusions This analysis suggests that keeping regular contacts with participants is probably the best approach to reduce refusals, which were, in this cohort, mainly determined by maternal sociodemographics characteristics.

\section{P1-255 TRENDS OF BRUCELLOSIS (MALTA FEVER) IN IRAN DURING THE PERIOD 1991-2008}

doi:10.1136/jech.2011.142976e.47

${ }^{1} \mathrm{E}$ Mostafavi, ${ }^{*} \mathrm{M}$ Asmand. ${ }^{1}$ Pasteur Institute of Iran, Tehran, Iran; ${ }^{2}$ Faculty of Veterinary Medicine, University of Tabriz, Tabriz, Iran

Introduction Brucellosis (Malta fever) is a bacterial zoonotic infectious disease. This study reviews the brucellosis trend in Iran during 1991-2008, provides the distribution maps related to the patient 